\title{
An Investigation of Human-Computer Interaction Approaches Beneficial to Weak Learners in Complex Animation Learning
}

\author{
Yu-Fang Yeh \\ Aletheia University, Taiwan
}

\begin{abstract}
Animation is one of the useful contemporary educational technologies in teaching complex subjects. There is a growing interest in proper use of learner-technology interaction to promote learning quality for different groups of learner needs. The purpose of this study is to investigate if an interaction approach supports weak learners, who have poor domain knowledge and comprehension difficulty of the learning subject, in complex animation learning. Three interaction approaches were designed and evaluated in an educational animation program teaching a complex subject of data structures. Participants were 70 undergraduate students performed poorly in the experimental course of introductory data structures. They were randomly assigned into one of the three interaction approaches: purereason-dialogue, predict-oriented, and reason-predict-combination interactions. Learning effects of these interaction approaches were measured by near-transfer and far-transfer tests as well as learning process surveys including perceived content difficulty, mental effort expenditure, and usefulness of the interaction approach. Findings indicate that the reasonpredict-combination interactions approach led to the greatest transfer performance and was rated by students as the most useful interaction approach for understanding the animation content. The findings generally recommend that for weak learners, interactions of reasoning dialogue is effective to develop near-transfer ability at the initial learning phase, whereas when learners' knowledge grows to be capable of near-transfer task, the predictoriented interactions become more helpful to gain far-transfer knowledge. Implications for design principles for interactive instructional animations and recommendations for future research are discussed.
\end{abstract}

Keywords: Human-computer interaction; Instructional animation; Weak learner; Data structures

\section{Introduction}

Nowadays, animation is considered as a useful instructional medium to depict complex subjects that involve extensive mental imagination for understanding such as cardiovascular system, meteorological phenomena, and computer data structures and algorithms. The educational power of animation is that it can help learners directly visualize temporal changes in state, shape and space of dynamic contents, and hence free up learners' mental resources from memorizing trivial object/event changes to performing important cognitive processes. 
Although animations have great educational potential, research evidence about their educational effectiveness is mixed. While some empirical studies show positive effects of animations on learning (Levy, Ben-Ari, \& Uronen, 2003; Yeh, Chen, Hung, \& Hwang, 2010). Other studies have found neutral effects (ChanLin, 1998; Morrison \& Tversky, 2001; Price, 2002) or even negative effects (Scheiter, Gerjets, \& Catrambone, 2006; Schnotz, Böckheler, \& Grzondzeil, 1999). One possible reason explaining no/negative effects might be that learners often are passive information receivers in animation learning, and tend not to actively reflect animation contents (Hundhausen, Douglas, \& Stasko, 2002).

To help learners taking advantages of animations, various interactions are used (Mayer \& Mareno, 2002). The animation-interactivity operation and reasoning dialogue-cannot only increase learning involvement and learning responsibility, but also trigger off necessary cognitive processes for understanding (Betrancout, 2005). To be effective, interactions must be well designed in accordance with learners' zone of proximal development (ZPD) (Vygotsky, 1978). The ZPD of a learner refers the knowledge and cognitive skills he/she can reach with interactions' assistance. Interactions that are not tailored to learner's ZPD, i.e. too simple to challenge new knowledge or too hard to respond, often results in "redundant effect" (Yeh et al., 2010), detracting learners from important cognitive processes.

In general, learners differ in ZPD. In addition, with learning progress, learners' ZPD may vary. Therefore, how interactions adjust its scaffolding strategy to timely stratify the varying ZPD of a learner is an important issue in educational technology research. Up to date, the design principles for dynamically adaptive to learner's varying ZPD remain unclear in literature on multimedia-animation learning and are needed more empirical studies. Therefore, this study intends to investigate how interactions can be made to better support learners from little cognitive skills to being more knowledgeable in animation learning. This study focused on dynamic interaction design for weak learners, who have little cognitive skills and have comprehension difficulty in the learning subject on their own, because they are especially sensitive to the interventions from interactions. Unlike knowledgeable learners have clear learning plans and are less affected by untailored interactions, weak learners largely depend on interactions to supplement their weak cognitive skills and scaffold them to cope with the learning challenge. For weak learners tailored interactions would make learning easier and effective, otherwise making learning more difficult. It is important to assess the impact of an interaction approach to weak learners.

\section{Effective Interactions in Educational Animations}

Interactions in forms of reasoning dialogue and predicting operations have been found significantly promoting animation learning (Byrne, Catrambone, \& Stasko, 1999; Yeh et al., 2010). Reasoning dialogue generally assists learners to propose reasons from the perspective of domain principles to support the animation actions and operations. The dialogue of interaction helps learners to relate animating content with its underlying domain rationales and principles. It assists learners to connect existing knowledge from schema with new knowledge presented in the animation and integrate them into a new, comprehensive knowledge structure of the learning subject. However, if learners are already able to spontaneously integrate related information into knowledge chunks, the external reason-dialogue interaction may become redundant (Sweller, 2005), consuming learner's limited mental resources on acquainted cognitive skills and thereby interfering learning. 
Accordingly, when learners already have general schema of the learning subject, the focus of interactions might better shift to verifying understanding. For example, guiding learners to sequentially predict what will be acted on the next scene of the animation might be a good method to diagnose the understanding about the animating content and stimulate the learner to amend misconceptions if any. In a predict-oriented interaction environment, the learner is prompted to predict operation(s)/event(s) going to act on the next scene of the animation. The predict-oriented interaction keeps on prompting the learner to predict upcoming events of the animation scene-by-scene until the learner makes an incorrect prediction. Once the learner makes a wrong prediction, the animation starts up to play out the correct operations and then prompt the learner to provide reasons to justify the animation's demonstration.

For weak learners learning with complex animations, previous research (Yeh et al., 2010) indicates that the reason-dialogue interaction is more beneficial than the predict-oriented interaction at the initial learning phase. The reason is when used at the initial phase of learning, the predict-oriented interaction is rather mental loading for weak learners because they usually have no sufficient knowledge to generate meaningful predictions. Instead, it mostly triggers weak learners to trial-and error or guessing, little helpful for deep understanding and systematic schema construction. However, with learning progress, weak learners may advance in cognitive skills and learning ability. Hence, the later phase of learner-animation interaction might better focus on motivating learners into generate procedure to-be-learned and verify their understanding learned at the previous phase. Whether the predict-oriented interaction approach is useful for weak learners at the later phase of animation learning is unknown.

In past research, the investigation of interaction effects to animation learning almost focus on the first animation practice at the initial learning phase of novice learners. However, students, especially weak learners, mostly practice with more than one animation before they thoroughly build the new knowledge to-be-learned. Therefore, it is important to explore what interaction approaches can effectively lead weak learners to systematic knowledge acquisition through a learning process involving multiple animation practices. To address the issue, the main purpose of this study is to investigate an interaction approach beneficial weak learners in an animation learning environment teaching a complex subject of data structures from the initial to the later animation practices. For the purpose, we compared three potentially useful interaction approaches: pure-reason-dialogue interaction, predict-orientated interaction, and reasonpredict-combination interaction, and examined which has the best effects to the entire animation learning of weak learners.

The study has three sub-purposes to answer the research questions that together make up the main purpose of this study. The three sub-purposes are:

(1) The study argues the reason-dialogue interaction and predict-oriented interaction have different instructional values. The former is beneficial for linking new knowledge with existing schema at the initial animation learning for weak learners, while the latter is beneficial at the later animation learning to verify understanding learned previously. Accordingly, the first subpurpose is to investigate if the reason-predict-combination interaction approach leads to greater learning outcomes than the other interaction approaches.

(2) It might be tiring to iteratively generate reasons already learned, consequently reducing learning motivation and learn less. Hence, the second sub-purpose is to investigate if the purereason-dialogue interaction approach motivates less mental effort investment and less perceived usefulness than the reason-predict-combination interaction approach in a whole. 
(3) An improper interaction strategy used at the beginning might negatively affect the entire learning process by inducing extraneous mental load, increasing processing difficulty, and interfering systemic knowledge construction. According, the third sub-purpose is to investigate if the predict-oriented interaction causes higher degrees of learner's perception on content difficulty and mental effort demand, and a less degree of usefulness perception.

\section{Methodology}

\section{Participants}

Participants were students enrolled in two classes of introductory data structures course of a university in Taiwan. They were taught by the same instructor. Because the target students of the current study were weak learners who had difficulty in comprehension of data structures, the instructor of the two participating classes determined students as weak learners of data structures based on impression of poor performance in classroom discussion and low scores in weekly quizzes and the mid-term exam. A total of 70 weak learners consisting of 51 males and 19 females with a mean age of 20.7 (range from 19 to 22) years old took part in this study. They volunteered to participate in this study for two extra credit points of the course. The large majority of the population was sophomores (97\%).

\section{Design}

The experiment was a between-subject design with participants randomly assigned into one of three experimental conditions: pure-reason-dialogue interaction $(n=24)$, predict-orientated interaction $(n=23)$, and reason-predict-combination interaction $(n=23)$. Each participant received two animations and two transfer tests and learning experience surveys. See Figure 1 for an overview of the design.

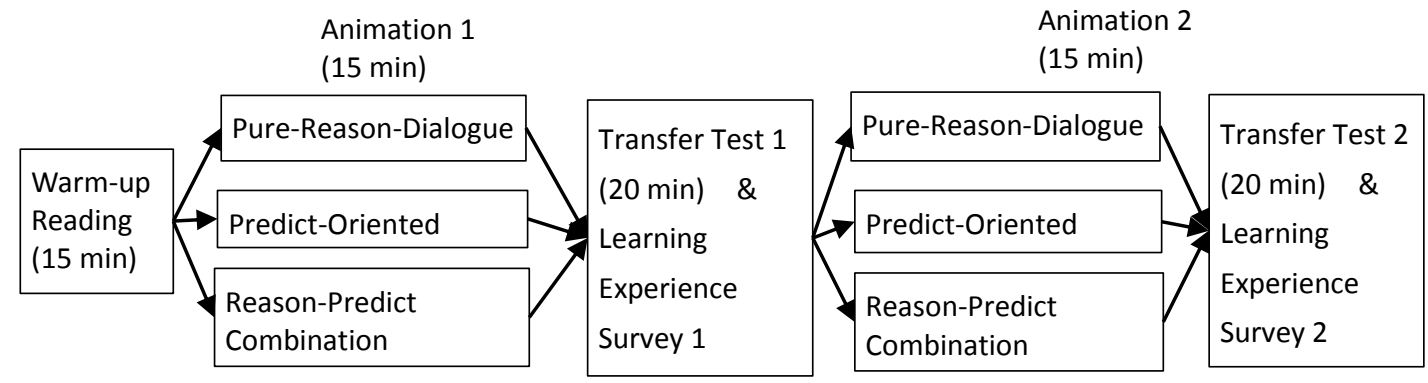

Figure 1. Overview of Experimental Design and Procedure

\section{The Animation Learning Program}

A web-based learning program consisted of two animations teaching AVL-tree insertion algorithm. Each animation was broken into four segments to allow learners to control animation presentation pace based on their own learning speed. The algorithm taught in the second 
animation was more complex than the first animation because of a greater amount of nodes and height of the tree. For the research purpose, three program versions were built. In all versions, the program delivered the same two narrative animations, yet only differed in interaction approaches. They were pure-reason-dialogue interactions, predict-oriented interactions, and reason-predict-combination interactions versions.

First, version pure-reason-dialogue interactions offered only one type of interactions -reasoning dialogue- through the two animations. In pure-reason-dialogue interactions version, the program segment-by-segment prompts learners to reason the algorithm acting on the animation by completing fill-in-the-blank reasoning statements.

Second, in version predict-oriented interactions, primary interactions focus on predicting algorithm operations up-playing in the following segment. In the predict-oriented interactions version, the program would go on prompting the learner to predict following animation actions until an incorrect prediction was made. Once incorrect prediction was made, the program plays correct algorithm procedures and prompts the learner to reason the animation just watched.

Third, the reason-predict-combination interactions version offered pure-reason-dialogue interaction in the first animation learning, and served predict-oriented interaction in the second animation learning. Figure 2 and 3 present two screenshots: one of the reason-dialogue interaction and one of the predict-oriented interaction.

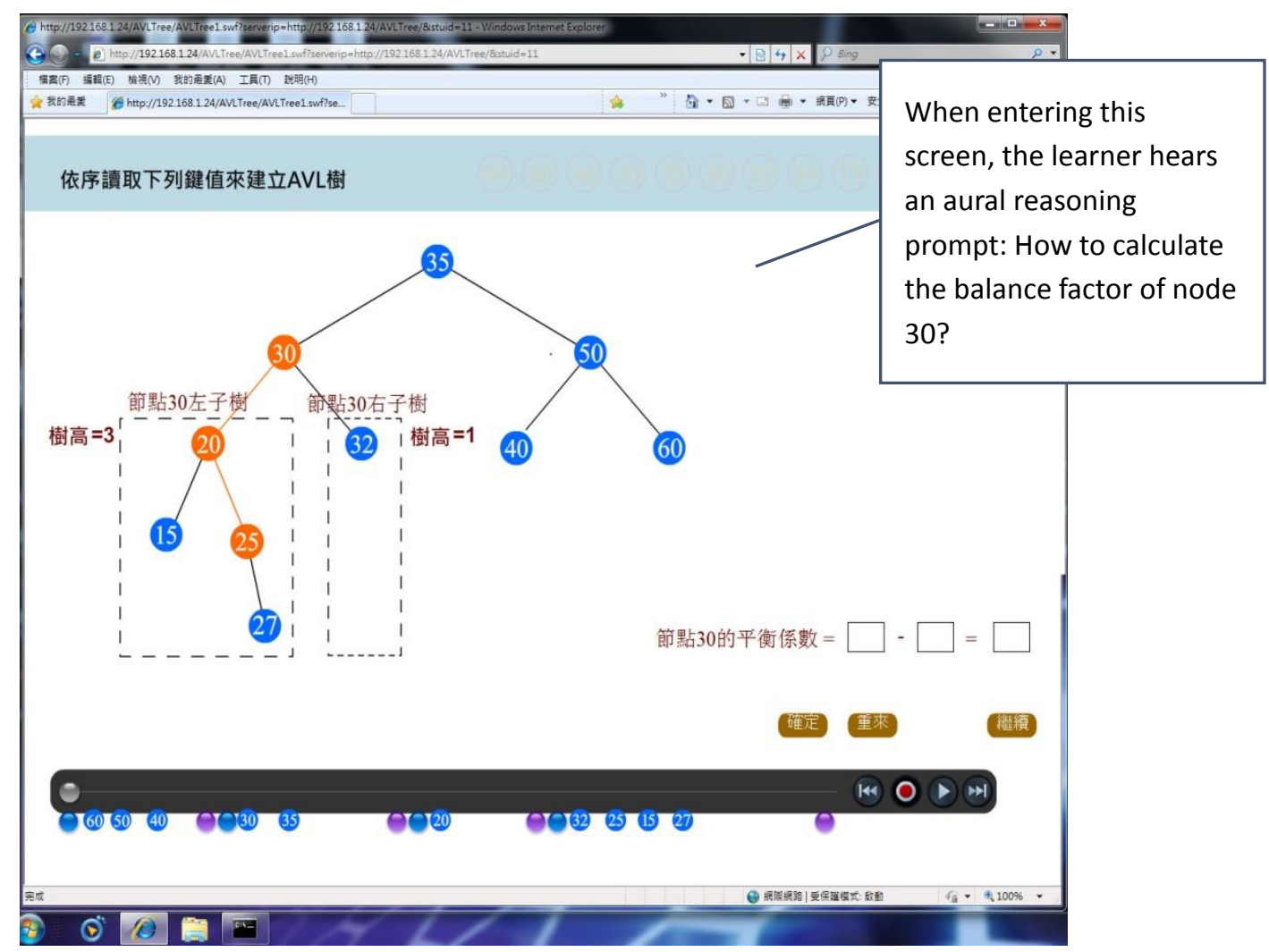

Figure 2. Screenshot of a Reason-dialogue Interaction 


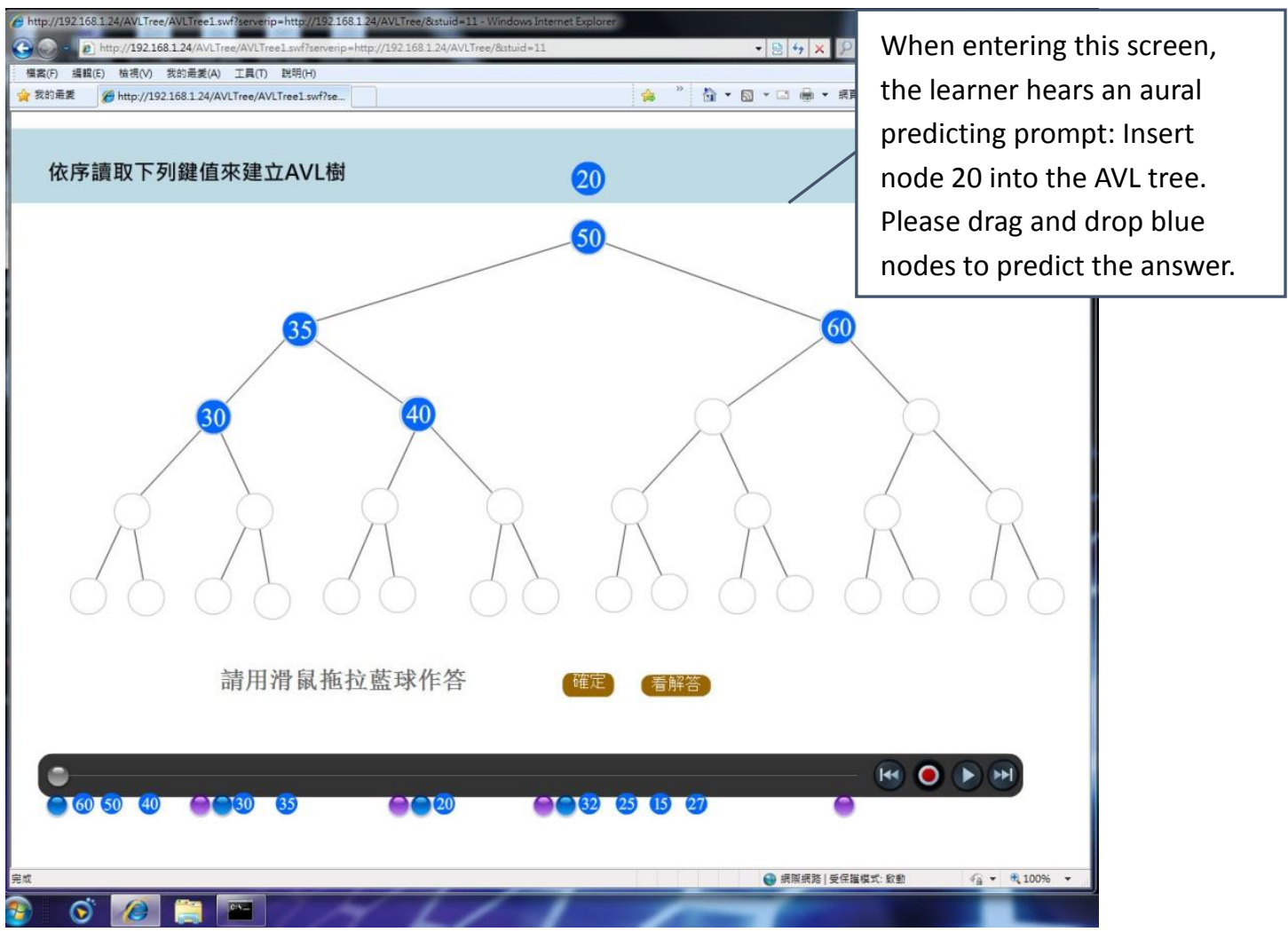

Figure 3. Screenshot of a Predict-oriented Interaction

\section{Measures}

\section{Knowledge Transfer Test}

To assess the extent of understanding the animations, two isomorphic knowledge-transfer tests were designed and administered right after the first and the second animations learning respectively. Both knowledge-transfer test consisted of a near-transfer question and a fartransfer question. The near-transfer question was similar to the example demonstrated in the animation. The far-transfer question was a question that required students to amend the algorithm taught in the animation in order to solve the given problem. Answers were scored as zero point for an incorrect answer or one point for a correct answer. Overall, a maximum of three points was achievable for the near-transfer question and far-transfer question, respectively. Examples of questions are as follows: 'What is the sum of balance factors of all nodes in the tree after the key adds into the tree?', 'What are the critical nodes that need to rotate to restore the property of AVL-tree', and 'Draw out the AVL tree after the insertions of following keys.' Questions in the first and the second knowledge-transfer tests were different in surface but isomorphic.

\section{Learning Process Survey}

Apart from learning outcomes, the quality of learning process is also important, which would affect learners' attitude to the subject and intention to re-use the program. The learning process 
quality was therefore surveyed. This study surveyed three aspects of learning process. They are learner's perceived content difficulty, mental effort invested for learning, and usefulness of the interactivity of the animation. The survey instrument consisted of three items, where one item measured one aspect of the learning process quality (see Table 1). The items measuring content difficulty and mental effort invested for learning were adapted from a widely used existing questionnaire of (Gerjets, Scheiter, Opfermann, Hesse, \& Eysink, 2009). For the instrument, students answered each item on a seven point Likert-type scale from 1 equaling 'extremely low' to 7 equaling, 'extremely high'.

Table 1. Items of the Learning Experience Questionnaire (Translated Version)

\begin{tabular}{ll}
\hline Content difficulty & $\begin{array}{l}\text { How easy or difficult do you consider AVL tree insertion } \\
\text { at this animation? }\end{array}$ \\
\hline Mental effort spent & $\begin{array}{l}\text { Indicate on the scale the amount of effort you exerted to } \\
\text { learn with the animation? }\end{array}$ \\
\hline Usefulness of interactions & $\begin{array}{l}\text { Indicate on the scale the extent of usefulness of the } \\
\text { interactions in this animation? }\end{array}$ \\
\hline
\end{tabular}

\section{Procedures}

The study consisted of three main steps. First, all participants were given 15 minutes to read a description of 443 words and three figures introducing what an AVL tree is and how AVL trees rotate to restore the height-balance property. The description was given to warm up students' prerequisite knowledge for the animation program. Second, all participants were randomly assigned into one of the three experimental conditions. Based on a pilot study, all participants were given 15 minutes to interact with each animation of their respective version of animation program. They were instructed to carefully study the animation because a test would be administered after completing one animation learning. Third, right completion of an animation learning, all students were asked to rate their animation learning experience, and took a knowledge transfer test. In total, two animations were learned, and the learning-experience surveys and knowledge-transfer tests were administered twice.

\section{Data Analysis}

Data were analyzed in five repeated-measures analyses of variance (ANOVA) to determine experimental effects on learning outcome and learning process with near-transfer score, fartransfer score, perceived difficulty of the learning content, mental effort investment, and perceived usefulness of the interaction as dependent variables, interaction approach (purereason-dialogue, predict-oriented, reason-predict-combination) as a between subjects variable, and time period (test 1 and test 2 ) as a within subjects variable. All tests of significance were run at the .05 level, indicating significant difference (Keppel, 1991).

\section{Findings}

The results are divided into two sections: learning transfer outcomes and learning process quality of the animation study. In the section of results of learning outcome tests, students' 
performance on near-transfer tests and far-transfer tests right after animation 1 and 2 learning were examined. The section of results of learning process survey reposts the effects of different interaction approaches on students' perceived difficulty of the animations, mental effort expenditure, and perceived usefulness of the interaction approach they received. Table 2 presents the relevant descriptive statistics among the three experimental groups regarding neartransfer scores, far-transfer scores, perceived difficulty of the animation, mental effort expenditure, and perceived usefulness of the interaction received. Furthermore, the results of post-hoc multiple comparisons with Bonferroni-method analysis were summarized in Table 3.

Table 2. Descriptive Statistics on Analysis of Animation Learning Effects among Different Interaction Approaches

\begin{tabular}{|c|c|c|c|c|}
\hline \multirow[t]{2}{*}{ Research variable } & \multicolumn{2}{|l|}{ Test 1} & \multicolumn{2}{|l|}{ Test 2} \\
\hline & Mean & SD & Mean & SD \\
\hline \multicolumn{5}{|c|}{ Pure-reason-dialogue interaction } \\
\hline Near-transfer score & 2.38 & .71 & 2.46 & .66 \\
\hline Far-transfer score & 1.25 & .74 & 1.25 & .79 \\
\hline Perceived difficulty & 5.29 & .91 & 3.83 & 1.24 \\
\hline Mental effort expenditure & 5.17 & 1.34 & 3.83 & .96 \\
\hline Perceived usefulness & 5.08 & 1.21 & 3.78 & 1.25 \\
\hline \multicolumn{5}{|l|}{$\begin{array}{l}\text { Reason-predict combination } \\
\text { interaction }\end{array}$} \\
\hline Near-transfer score & 2.26 & .69 & 2.52 & .73 \\
\hline Far-transfer score & 1.22 & .85 & 2.09 & .90 \\
\hline Perceived difficulty & 5.39 & .99 & 5.17 & .93 \\
\hline Mental effort expenditure & 5.21 & 1.13 & 5.43 & 1.04 \\
\hline Perceived usefulness & 5.04 & 1.15 & 5.70 & .97 \\
\hline \multicolumn{5}{|l|}{ Predict-oriented interaction } \\
\hline Near-transfer score & 1.48 & .90 & 2.09 & .85 \\
\hline Far-transfer score & 0.91 & .60 & 1.17 & .98 \\
\hline Perceived difficulty & 6.26 & .75 & 5.65 & .93 \\
\hline Mental effort expenditure & 5.90 & .81 & 5.78 & 1.09 \\
\hline Perceived usefulness & 3.35 & 1.11 & 3.87 & 1.18 \\
\hline
\end{tabular}

\section{Results of Learning Outcome Tests}

Using repeated-measures ANOVA, a significant difference was identified in the near-transfer mean score, $F(2,67)=7.63, \mathrm{MSE}=5.98, p=0.001$, among students of the three different interaction conditions. A post-hoc multiple comparisons with Bonferroni method analysis showed that students in the conditions pure-reason-dialogue interaction and reason-predictcombination interaction had much higher near-transfer performance scores than students in the condition predict-orientated interaction ( $p=0.003$ and $p=0.005$, respectively).

Regarding far-transfer performance, the result of repeated-measures ANOVA revealed there exists significant effect of the animation interaction approach, $(F(2,67)=5.34$, MSE $=4.41, p=$ $0.007)$. Bonferroni-method post-hoc tests revealed that students in the condition reasonpredict-combination interaction had higher far-transfer performance scores than students in the condition predict-orientated interaction $(p=0.006)$. 
The result of post-hoc comparisons on the learning outcome scores suggests that reason-predictcombination interaction is a better interaction design in animation learning because it lead students to superior near- and far-transfer performance.

\section{Results of Learning Process Surveys}

Through repeated measures ANOVA, significant differences were found in mean perceived animation difficulty $(F(2,67)=25.92$, MSE $=22.85, p<0.000)$, mental effort expenditure $(F(2$, $67)=12.29, \mathrm{MSE}=21.14, p=0.000)$, and perceived usefulness of the interaction $(F(2,67)=29.72$, MSE $=35.70, p=0.000$ ) among participants in the three experimental conditions as operationalized in the study.

Bonferroni-method post-hoc tests demonstrated that participants in predict-oriented interaction condition rated higher perceived animation difficulty than those in pure-reason-dialogue interaction $(p=0.000)$ and in reason-predict-combination interaction $(p=0.003)$ conditions, and participants in reason-predict-combination interaction condition had higher perceived animation difficulty than those in the pure-reason-dialogue interaction condition $(p=0.001)$. Regarding mental effort expenditure, the reason-predict-combination interaction and the predict-oriented interaction groups rated higher degrees than the pure-reason-dialogue interaction group ( $p=$ 0.01 and $p=0.000$, respectively). In terms of perceived usefulness of the interaction, the reasonpredict-combination interaction group rated higher usefulness degrees than the pure-reasondialogue interaction $(p=0.000)$ and the predict-oriented interaction $(p=0.000)$ groups, and the pure-reason-dialogue interaction group rated higher than the predict-oriented interaction group $(p=0.001)$.

The result of post-hoc comparisons regarding to the learning process variables implies that reason-predict-combination interaction in general is superior to the other two interaction approaches because of the highest perceived usefulness of interaction, lower perceived content difficulty than predict-oriented interaction, and more mental effort involvement than purereason-dialogue interaction.

Table 3. Post-hoc Multiple Comparisons

\begin{tabular}{|c|c|c|c|c|c|c|c|c|}
\hline \multirow{2}{*}{$\begin{array}{l}\text { Dependent } \\
\text { variable }\end{array}$} & \multicolumn{2}{|c|}{ interaction } & \multirow{2}{*}{$\begin{array}{l}\text { Mean } \\
\text { difference } \\
(I-J)\end{array}$} & \multirow{2}{*}{$\begin{array}{l}\text { Std. } \\
\text { error }\end{array}$} & \multirow[t]{2}{*}{ Sig. } & \multicolumn{2}{|c|}{$\begin{array}{l}95 \% \text { Confidence } \\
\text { interval }\end{array}$} & \multirow{2}{*}{$\begin{array}{l}\text { Post hoc } \\
\text { analysis }\end{array}$} \\
\hline & (I) & (J) & & & & $\begin{array}{l}\text { Lower } \\
\text { bound }\end{array}$ & $\begin{array}{l}\text { Upper } \\
\text { bound }\end{array}$ & \\
\hline \multirow{3}{*}{$\begin{array}{l}\text { Near } \\
\text { transfer }\end{array}$} & $\mathrm{RP}$ & $\mathrm{R}$ & -.0254 & .18256 & 1.000 & -.4736 & .4229 & \\
\hline & $\mathrm{RP}$ & $P$ & $.6087^{*}$ & .18449 & .005 & .1557 & 1.0617 & $\mathrm{RP}>\mathrm{P}$ \\
\hline & $\mathrm{R}$ & $\mathrm{P}$ & $.6341^{*}$ & .18256 & .003 & .1858 & 1.0823 & $R>P$ \\
\hline \multirow[t]{3}{*}{ Far transfer } & $\mathrm{RP}$ & $\mathrm{R}$ & .4022 & .18753 & .107 & -.0583 & .8627 & \\
\hline & $\mathrm{RP}$ & $P$ & $.6087^{*}$ & .18952 & .006 & .1433 & 1.0741 & $\mathrm{RP}>\mathrm{P}$ \\
\hline & $\mathrm{R}$ & $\mathrm{P}$ & .2065 & .18753 & .824 & -.2540 & .6670 & \\
\hline \multirow{3}{*}{$\begin{array}{l}\text { Perceived } \\
\text { difficulty }\end{array}$} & $\mathrm{RP}$ & $\mathrm{R}$ & $.7201^{*}$ & .19371 & .001 & .2444 & 1.1958 & $\mathrm{RP}>\mathrm{R}$ \\
\hline & RP & $P$ & $-.6739 *$ & 19576 & .003 & -1.1546 & -.1932 & $\mathrm{RP}<\mathrm{P}$ \\
\hline & $\mathrm{R}$ & $\mathrm{P}$ & $-1.3940 *$ & 19371 & .000 & -1.8697 & -.9184 & $\mathrm{R}<\mathrm{P}$ \\
\hline \multirow{3}{*}{$\begin{array}{l}\text { Mental } \\
\text { effort }\end{array}$} & $\mathrm{RP}$ & $\mathrm{R}$ & $.8261^{*}$ & .27057 & .010 & .1617 & 1.4905 & $\mathrm{RP}>\mathrm{R}$ \\
\hline & $\mathrm{RP}$ & $P$ & -.5000 & .27344 & .216 & -1.1714 & .1714 & \\
\hline & $\mathrm{R}$ & $P$ & $-1.3261^{*}$ & .27057 & .000 & -1.9905 & -.6617 & $\mathrm{R}<\mathrm{P}$ \\
\hline
\end{tabular}




\begin{tabular}{lllllllll}
\hline Perceived & $\mathrm{RP}$ & $\mathrm{R}$ & $.9321^{*}$ & .22615 & .000 & .3767 & 1.4874 & $\mathrm{RP}>\mathrm{R}$ \\
usefulness & $\mathrm{RP}$ & $\mathrm{P}$ & $1.7609^{*}$ & .22854 & .000 & 1.1997 & 2.3221 & $\mathrm{RP}>\mathrm{P}$ \\
& $\mathrm{R}$ & $\mathrm{P}$ & $.8288^{*}$ & .22615 & .001 & .2735 & 1.3841 & $\mathrm{R}>\mathrm{P}$ \\
\hline
\end{tabular}

Note: $\mathrm{R}=$ pure-reason-dialogue; $\mathrm{RP}=$ reason-predict-combination; $\mathrm{p}=$ predict-oriented.

*The mean difference is significant at the .05 level.

\section{Discussion and Conclusions}

This study investigated how interactions can be made more beneficial through their adaptation to students' progress in cognitive skills. This study designed three interaction approaches and implemented an experiment to test which interaction approach is most beneficial for weak learners in a complex animation learning context such as instructing data-structures. This study found that the interaction approach reason-predict-combination led weak learners to the greatest learning-transfer performance and was rated by weak learners the most useful for helping understanding the animation content, though it did not significantly reduce perceived content difficulty and mental effort demand as compared to other interaction approaches. These results could be interpreted that that reason-predict-combination interaction dynamically adjusted interaction strategy to fit into the knowledge level and learning potential of the learner, maintained the interactive activities on a challengeable level (not too easy to get tired of the interaction and not too difficult to respond to the interaction) so that it successfully encouraged the learner keeping investing effort on refining their cognitive skills, hence resulting in superior learning outcomes to their counterparts instructed by other interaction approaches.

The results also indicate that the pure-reason-dialogue interaction led to good near-transfer performance but had little effects on far-transfer learning. Weak learners receiving pure-reasondialogue interaction in the first animation learning had superior near-transfer performance to their counterparts in the predict-oriented interaction condition. However, weak learners who continued receiving pure-reason-dialogue interaction in the second animation learning learned little, no significant improvement on both near- and far-transfer scores. Additionally, data of the learning process surveys indicate that students in the pure-reason-dialogue interaction condition perceived less content difficulty and spent less mental effort in the second animation learning than they did in the first animation learning. An explanation for the fading effect of the purereason-dialogue interaction approach might be that fixed interactive activities might lose students' motivation and attention to the learning content so that they ignored the diversity between animations and learned in a cognitive economy way (less mental effort investment), consequently little knowledge acquisition.

Furthermore, the current study found that the predict-oriented interaction approach was least effective for weak learners through the entire animation learning phases. Students in the predictoriented interaction condition performed poorest in both near-and far-transfer tests, and students reported that predicting tasks were very mental demanding, they increased learning difficulty, and they were least useful for assisting understanding. These findings are in line with Yeh et al.'s (2010) findings that predict-oriented interaction approach is not a good teaching strategy for learners with weak knowledge base of the learning subject. The current study further indicates that weak learners hardly not benefit from predict-oriented interaction because they usually have not enough knowledge to perform meaning prediction, at the most time the predicting task only bring about trial-and-error guessing, little help for deep understanding. The predict-oriented interaction promoted cognitive skills development only if the learner had solid fundamental knowledge about the subject. This claim was supported by the current finding that 
students who had good near-transfer scores received predict-oriented interaction had superior far-transfer performance to their counterparts continuing receiving pure reasoning tasks.

This study contributes to interactive instructional animation research by providing empirical evidences that an interaction approach varied along with learning progress is superior to fixed, single-strategy interaction approaches. This study specifically suggests a varied interaction approach useful for weak learners in complex animation learning. The findings imply that an interaction strategy is not absolutely useful or unhelpful. It depends on when to use to whom. An interaction strategy useful at the initial learning phase is possibly fading its effectiveness at the later learning phase. On the other hand, interactions un-tailored to the learner at the initial animation learning might be useful when being applied at the later learning phase.

A practical implication for instructional designers is that it is not necessary to make the animation to fully narrate and act out the entire instructional content; the design should focus on creating an interactive environment where the learner and the animation can work together to cooperatively generate the to-be-learned knowledge. Although varied interactions have been recognized a useful instructional strategy, an interaction strategy should not be randomly selected and delivered to the learner, they should be served in accordance with learning progress in order to better motivate learners to continuously invest effort to learn, and help learners systemically develop near transfer and far transfer abilities.

Finally, there are some possible limitations to the current study that should be addressed in the future research. Meanwhile, the current study assessed interaction approached in a subject, which might limit the generalizability of the research findings. It is suggested that more educational contexts be experimented to provide additional empirical evidence. In addition, in the current study, the time on interacting with an animation was limited in 15 minutes. The interaction effects may be different when the learners have more time to interact with each animation, or when the learner has the free to control the interacting time on one animation. Future study should address the issue of interacting time. Moreover, only three interaction approaches were compared in the current study. Future research could be conducted to examine more interaction approaches in effects of cognitive skills development and learning process variables for different characteristics of learners.

\section{References}

Betrancout, M. (2005). The animation and interactivity principles in multimedia learning. In R. E. Mayer (Ed.), The Cambridge handbook of multimedia learning (pp. 287-296). Cambridge, UK: Cambridge University Press.

Byrne, M. D., Catrambone, R., \& Stasko, J. T. (1999). Examining the effects of animation and predictions in student learning of computer algorithms. Computers \& Education, 33, 253278.

ChanLin, L. J. (1998). Animation to teach students of different knowledge levels. Journal of Instructional Psychology, 25, 166-175.

Gerjets, P., Scheiter, K., Opfermann, M., Hesse, F. W. \& Eysink, T.H.S. (2009). Learning with hypermedia: The influence of representational formats and different levels of learner control on performance and learning behavior. Computers in Human Behavior, 25, 360370. 
Hundhausen, C. D., Douglas, S. A., \& Stasko, J. T. (2002). A meta-study of software visualization effectiveness. Journal of Visual Languages and Computing, 13(3), 259-290.

Keppel, G. (1991). Design and analysis: A researcher's guide. Upper Saddle River, NJ: Prentice Hall.

Levy, R.B.-B., Ben-Ari, M., \& Uronen, P.A. (2003). The Jeliot 2000 program animation system. Computers \& Education, 40(1), 1-15.

Mayer, R. E., \& Moreno, R. (2002). Animation as an aid to multimedia learning. Educational Psychology Review, 14, 87-99.

Morrison, J., \& Tversky, B. (2001). The (in)effectiveness of animation in instruction. In J. Jacko \& A. Sears, Eds. Extended Abstracts of the ACM Conference on Human Factors in Computing Systems, pp. 377-378. Seattle: ACM.Park, O. C., \& Gittelman, S. S. (1992). Selective use of animation and feedback in computer-based instruction. Educational Technology, Research and Development, 40, 125-167.

Price, S. J. (2002). Diagram representation: The cognitive basis for understanding animation in education (Technical Report CSRP 553): School of Computing and Cognitive Sciences, University of Sussex.

Scheiter, K., Gerjets, P., \& Catrambone, R. (2006). Making the abstract concrete: Visualizing mathematical solution procedures. Computers in Human Behavior, 22, 9-25.

Schnotz, W., Böckheler, J., \& Grzondzeil, H. (1999). Individual and co-operative learning with interactive animated pictures. European Journal of Psychology of Education, 14, 245-265.

Sweller, J. (2005). The redundancy principle in multimedia learning. In R. E. Mayer (Ed.), The Cambridge handbook of multimedia learning (pp. 159-167). Cambridge, UK: Cambridge University Press.

Vygotsky, L. S. (1978). Mind in society: The development of higher psychological process. Cambridge, M S: Harvard University Press.

Yeh, Y.-F., Chen, M.-C., Hung, P.-H., \& Hwang, G.-J. (2010). Optimal self-explanation prompt design in dynamic multi-representational learning environments. Computers \& Education, 54, 1089-1100.

Correspondence: Yu-Fang Yeh, Assistant Professor, Sports Information and Communication, Aletheia University, New Taipei City, Taiwan 